\title{
Variations in body shape of European seabass larvae (Dicentrarchus labrax) reared under xenic and axenic conditions
}

\author{
Eyasu Shumbulo Shuba ${ }^{* 1}$, Dominique Adriaens ${ }^{2}$ and Peter Bossier ${ }^{2}$ \\ ${ }^{*}$ Arba Minch University P.O.Box 21, Arba Mich, Ethiopia, E-mail: eyasu78@yahoo.com Tel: +251-923-026699 \\ ${ }^{2}$ Ghent University Sint-Pietersnieuwstraat 25, B - 9000 Ghent, Belgium
}

\begin{abstract}
High rates of body deformities is one of the most challenging problems in fish culture and is a common source of downgrading the product value of commercially raised fish. The aims of this study are explore the effects of xenic and axenic rearing conditions on the seabass larvae and check whether the rearing condition has a different effect as larvae grow older. The larvae of seabass larvae, Dicentrarchus labrax L. (Teleostea, Moronidae), were reared under xenic and axenic rearing conditions up to fourteenth day after hatching (DAH). The size and shape were analyses for specimens of three age groups: DAH zero, four and 12. The growth comparison was done based on length measurements. For specimens of DAH zero and four, the total length, gut length, predorsal fin length, yolk sac length and height were measured. For specimens of DAH 12 the total length, standard length, gut length, dorsal fin fold length and height, anal fin fold length and height, preanal fin fold length, predorsal fin fold length, eye diameter, head height, hind gut length and notochord diameter were measured. The average measurements for these metric variables were lower in axenic specimens than xenic ones suggesting better performance in xenic treatment. The variations in body shape were studied and quantified using geometric morphometrics. The results showed significant differences in shape between the xenic and axenic specimens at all age groups. Thus, the importance of larval rearing conditions in determining seabass shape and quality is evident.
\end{abstract}

Key words/phrases: seabass, aquaculture, body deformity, geometric morphometrics DOI: http://dx.doi.org/10.4314/ejst.v7i2.2

\section{INTRODUCTION}

The seabass, Dicentrarchus labrax, Linnaeus 1758, is primarily marine fish that sometimes enters brackish and freshwaters, tolerating wide range of environmental conditions: eurythermic $\left(5-28^{\circ} \mathrm{C}\right)$ and euryhaline (3\% to full strength sea water). Historically it was cultured in coastal lagoons and tidal reservoirs before the mass-production of juveniles started in the late 1960s (FAO, 2007). Of all non-salmonid species, the seabass was the first marine species to be commercially cultured in Europe and at present it is the most important commercial fish widely cultured.

The high rates of occurrence of body deformities is one of the most challenging problems in fish culture and are a common source of downgrading the product value of commercially raised fish (Sfakianakis et al., 2006; Verhaegen et al., 2007). Various sources of body deformities have been identified: pollutants (Bengtsson et al., 1988; Nguyen and Jansen, 2002), temperature (Polo et al., 1991), water currents (Divanach et al., 1997), nutrition (Dedi et al., 1995; Takeuchi et al., 1998; Haga et al., 2002), diseases (Breinholt and Heckmann, 1976), and genetics (Campbell, 1995). In general, according to Sfakianakis et al. (2006) they develop due to the lack of sufficient knowledge on the optimum environmental requirements of fish at the different stages of their life. Despite the impressive progress made in the rearing methods, nutrition and disease control, morpho-anatomical abnormalities continue to affect the hatchery production (Koumoundouros et al., 2002, Koumoundouros

\footnotetext{
*Corresponding author

(C) This is an Open Access article distributed under the terms of the Creative Commons Attribution License (http://creativecommons.org/licenses/CC BY4.0)
} 
et al., 2004, Planas and Cunha, 1999, Boglione et al., 2003, Cahu et al., 2003 and Sfakianakis et al., 2004). They mainly develop during the early life history of fish, and can affect external morphology, survival, growth rate, behavior and finally production cost and effectiveness of the hatcheries (Divanach et al., 1996, Koumoundouros et al., 1997).

This study was intended to explore the effects of xenic and axenic rearing conditions on the seabass larvae in order to reveal the impact of these on the shape of the fish. Moreover, it was designed to see whether the rearing condition has a different effect as larvae grow older.

\section{MATERIALS AND METHODS}

\section{Rearing conditions}

Eggs were brought from Gravelines, France in big plastic bag and transferred to 20 liter tank. In this tank, the eggs were acclimatized and portion of the eggs were disinfected ( 3 minutes in $100 \mathrm{ppm}$ gutaraldehyde, 2400 eggs/L) for axenic set ups, so at this point there were xenic and axenic eggs. The disinfected ones were stored in incubation bottles for axenic rearing and the remaining eggs were kept in 20 liter tank for xenic treatment. From each treatment, 2 x 96 eggs are individually stored in two 96 multi-well plates: for this, eggs from one incubation bottle are taken. In this way, all eggs are exactly in the same medium, thus reducing the variation within a treatment. The eggs that remained in the tank are kept in a $20 \mathrm{~L}$ tank. The $20 \mathrm{~L}$ tank with UV sterilized water at $16^{\circ} \mathrm{C}$ was put on top of table for continuous flow-through and the tank was refilled several times a day. Microbiology test was done on samples $24 \mathrm{hrs}$ after disinfection. This is performed on thirty eggs taken from the first eight bottles of incubation, homogenised and plated. To check tubes for contamination, a sample of one $\mathrm{ml}$ was taken from each incubation bottle and added to a sterile tube containing $9 \mathrm{ml}$ marine broth (10\%). After third day of incubation, the eggs hatched and stocking was done as of three o'clock in the morning of this day.

The larvae were reared under two setups: axenic and xenic. Twelve larvae from each axenic, i.e. the larvae from the disinfection, and xenic were stocked in vials containing $10 \mathrm{ml}$ of sterile seawater. Xenic larvae are taken from the tank. Axenic larvae are taken from bottles. From each bottle that is used for stocking the larvae in the vials, 10 larvae were homogenised and plated on $10 \%$ marine agar (MA) to check for axenity. The larval rearing continued up to the $14^{\text {th }}$ day.

\section{Samples}

A total of 45 samples were taken for each sampling dates. The larvae were anaesthetized with Tricaine methanesulfonate, (TMS or MS-222) and kept in $8 \%$ formalin. After 72 hours the specimens were transferred to $70 \%$ ethanol for storing.

\section{Image acquisition}

Images were taken using the following procedures:

The specimen was taken with plastic dropper and placed on a glass slide prepared for this purpose by applying hot wax with the use of metal frame heated by Bunsen flame (Figure1). The specimen was washed three times with distilled water while it was on the slide. To this slide with the specimen, a drop or two of $1.6 \%$ alizarin solution was added and gently shacked for staining. This was washed three times again with distilled water to remove the remains of alizarin. At this time the right positioning of the larvae was checked under a light microscope and nail polish applied to it to fix the position. The specimen stained with alizarin and fixed with nail polish was placed in a series of baths starting from a bath of distilled water to the baths of glycerin at four different concentrations (i.e. $25 \%, 50 \%, 75 \%$ and $100 \%$ ) for 30 minutes in each. At the end of bathing period, drops of $100 \%$ glycerin were added to the 
slides with stained and fixed specimen, and covered with cover glass by applying heat from a hot metal frame. Finally, images were taken with a digital camera (colorveiw 8 SIS) attached to a microscope (Olympus 52x9) at 25X magnification with a lens of $0.5 \mathrm{X}$ magnification.

\section{Length measurements}

For length measurements of specimens from DAH zero and DAH four, TpsDig software was used (Rohlf, 2012). For this software the tps file was created by using tps file utility program. TpsDig was used for digitizing landmarks for geometric morphometric analyses (Figure 2). For DAH 12 specimens ImageJ software was used to measure the lengths. For specimens of DAH 0 and 4, five measurements were taken (Figure 3): total Length (TL), gut length (GL), yolk sac length (YSL), yolk sac height (YSH), and predorsal fin length (PDL). The yolk sac size was calculated based on the formula of ellipse area.

For specimens of DAH 12, a total of 13 measurements were taken (Figure 3) comprising the total length (TL), standard length (SL), gut length (GL), dorsal fin fold length (DFFL), anal fin fold length (AFFL), preanal fin fold length(PAFFL), predorsal fin fold length (PDFFL), eye diameter (ED), head height (HH), hind gut length (HGL), notochord diameter (ND), dorsal fin fold height (DFFH) and anal fin fold height (AFFH).

For shape analyses the elliptic Fourier software was used (Iwata, 2010). This software extracts the contour shape from a full color bitmap image, delineates the contour shape with the elliptic Fourier descriptors, and performs a principal component analysis for summarizing the shape information. The original JPG image contours were redrawn manually in corel draw and saved as bitmap file (Figure 4). This was done in order to prepare files for loading in elliptic Fourier software so that appropriate contours were considered by the software.
The elliptical Fourier analysis was used to fit specimen outlines. A PCA was performed for each new data set. The first three principal component axes were plotted against total length as majority of variation is explained by the first three PCs.

\section{Statistical analyses}

The mean, standard deviation, minimum and maximum were calculated from original length data in $\mathrm{mm}$. The original length data were converted to log transformed data in order to see the linear relationships between variables. The regression analyses were performed for log transformed length data to check for correlation and pattern of growth in the metric variables. The ANOVA and MANOVA were done to check for statistical variation between groups. The principal component analyses (PCA) were performed to see the pattern of variation that exists. The CVA scatter plot was given for lengths measurements to identify the measured variable that best explains the differences among the treatment groups.

\section{RESULTS}

\section{Growth analyses}

While the total length of larvae increased by only $17 \%$ in axenic treatments at day 12 post-hatching, the increase was up to $33 \%$ in xenic ones. The average value obtained for total length, gut length, yolk sac length and height are higher in xenically treated larvae whereas the average predorsal length was higher in axenically treated larvae at DAH 0 and 4 (Table 1). To test if these differences are statistically significant, an ANOVA was performed. Accordingly, the axenic larvae have a significantly smaller total length $\left(\mathrm{F}_{1,46}=17.59, \mathrm{p}<0.01\right)$, gut length $\left(\mathrm{F}_{1}\right.$, $\left.{ }_{46}=37.89, \mathrm{p}<0.01\right)$, yolk sac length $\left(\mathrm{F}_{1,46}=28.64\right.$, $\mathrm{p}<0.01)$, and yolk sac height $\left(\mathrm{F}_{1,46}=17.59, \mathrm{p}\right.$ $<0.01)$. However, the predorsal length was significantly higher in axenically treated larvae $\left(\mathrm{F}_{1,46}=\right.$ $18.32, \mathrm{p}<0.01)$. 


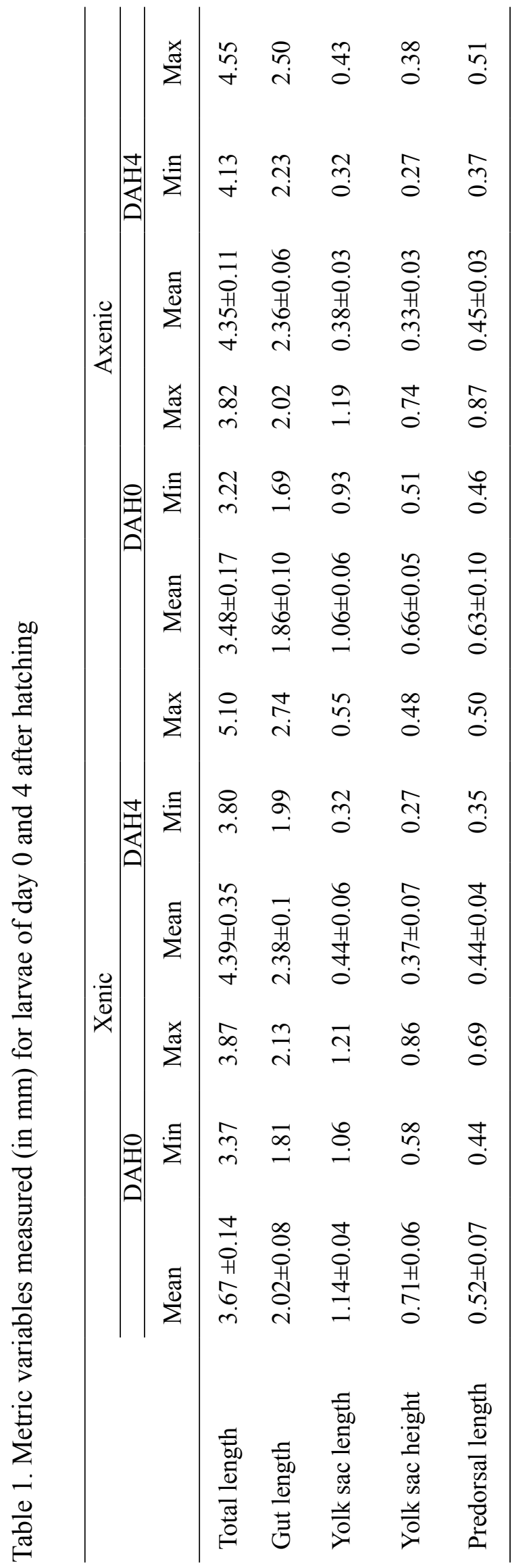

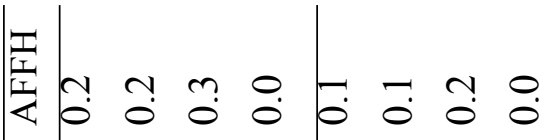

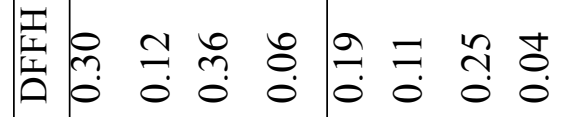

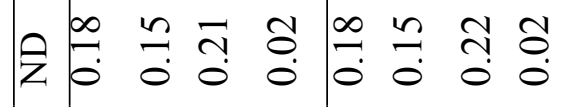

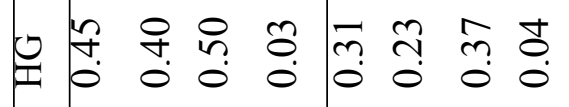

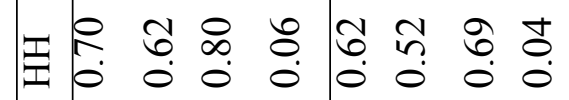

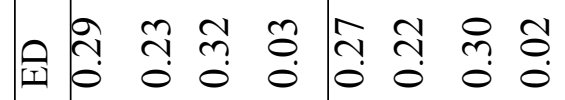

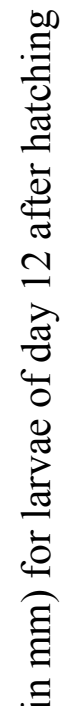

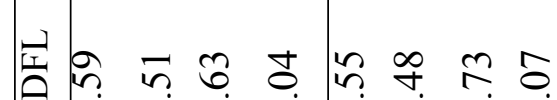

है

हี

$\frac{50}{5}$

II

可

t.ํำ

ב

可

严

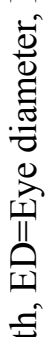

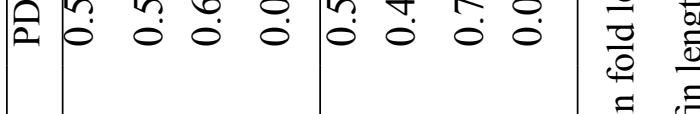

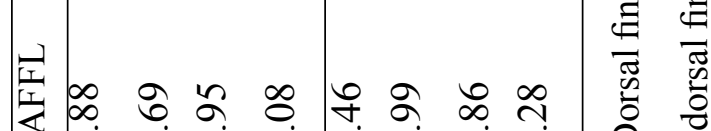

死

i⿱

武

$\begin{array}{llllllll}4 & 4 & 4 & 0 & -1 & - & 0\end{array}$

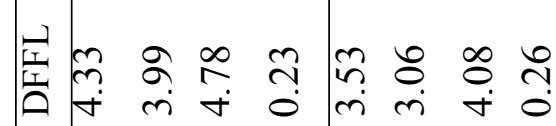

응

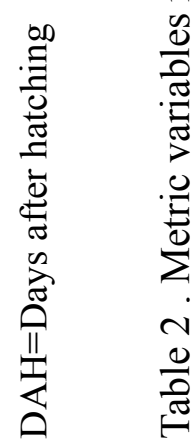

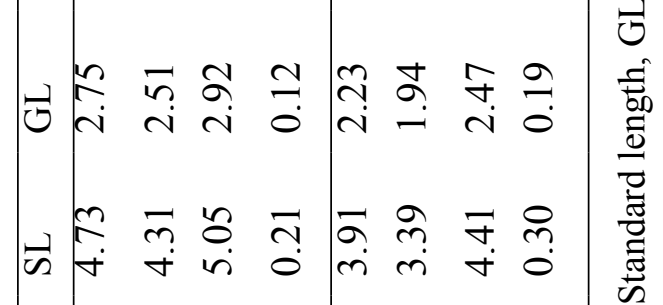

퓨융

递

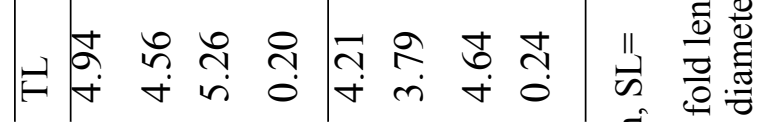

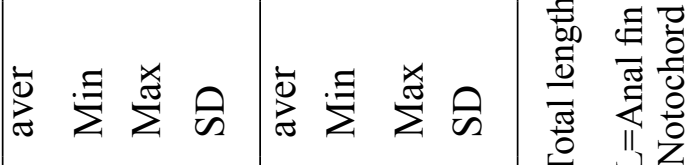

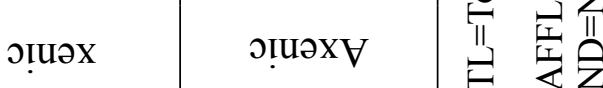


At day 12 after hatching, axenic larvae have smaller average values (Table 2) for almost all metric variables measured. The ANOVA test confirmed the significant differences for all variables measured except for the diameter of eye $\left(\mathrm{F}_{1,34}=2.87, \mathrm{p}>0.01\right)$ and the diameter of notochord $\left(\mathrm{F}_{1,34}=0.27, \mathrm{p}>0.01\right)$.

The log transformed data of several metric variables over total length measurements of xenic and axenic specimens of DAH 4 were used for the purpose of treatments except the yolk sac height which is negatively allometric in axenic specimens (slope $=-1.06)$.

The differences in the metric variables among the two treatments were statistically significant in all age groups as tested by MANOVA $\left(\mathrm{F}_{1,46}=86.32, \mathrm{p}<0.01\right),\left(\mathrm{F}_{1,49}=31, \mathrm{p}<0.01\right)$ and $\left(\mathrm{F}_{1,34}=501.1, \mathrm{p}<0.01\right)$ in DAH0, DAH4 and DAH12, respectively. The CVA scatter plot
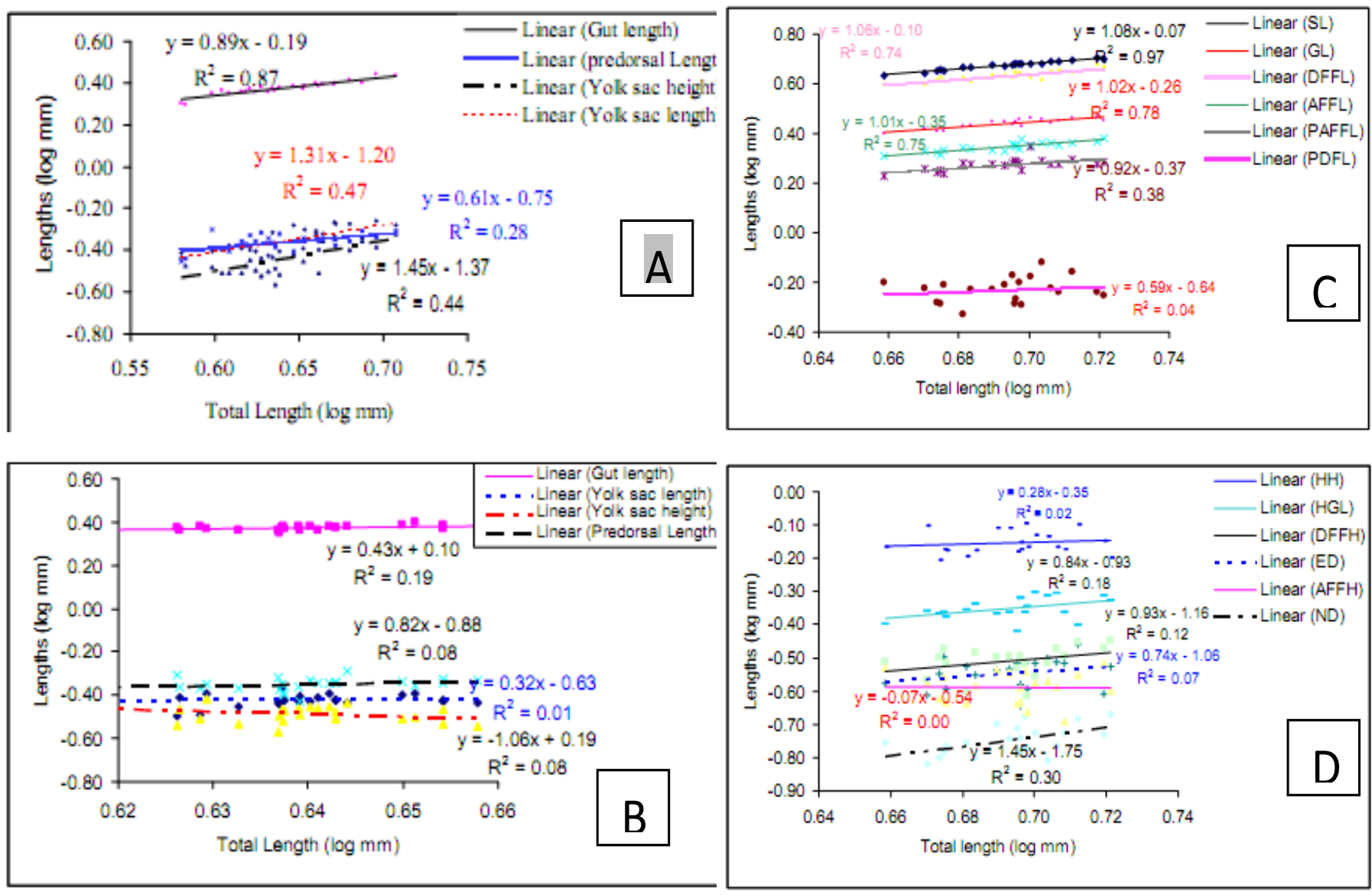

$\mathrm{A}=$ xenic DAH 4; $\mathrm{B}=$ Axenic DAH4; $\mathrm{C}=$ xenic DAH12 and $\mathrm{D}=\mathrm{Axenic} \mathrm{DAH} 12$

Figure 1. Plot of log transformed data of some metric variables over total length measurements

comparison. The gut length exhibited a strong positive correlation $\left(\mathrm{R}^{2}=0.85\right)$ with respect to total length in xenic larvae of day 4 after hatching but the case is not true for axenically treated larvae. In xenic specimens the gut growth exhibited isometric growth patterns with the slope of the regression line 0.89 , whereas negatively allometric in axenic larvae. Other metric variables displayed positive allometric growth pattern (Figure 1) with total length in both
(Figure 2) for length data for both DAH0 and DAH4 reveals that the predorsal and yolk sac length are better discriminators between the xenic and axenic groups. But for day 12 after hatching total length, anal fin fold and hind gut length are better discriminators between the two treatments as revealed by the CVA scatter plot (Figure 2). 

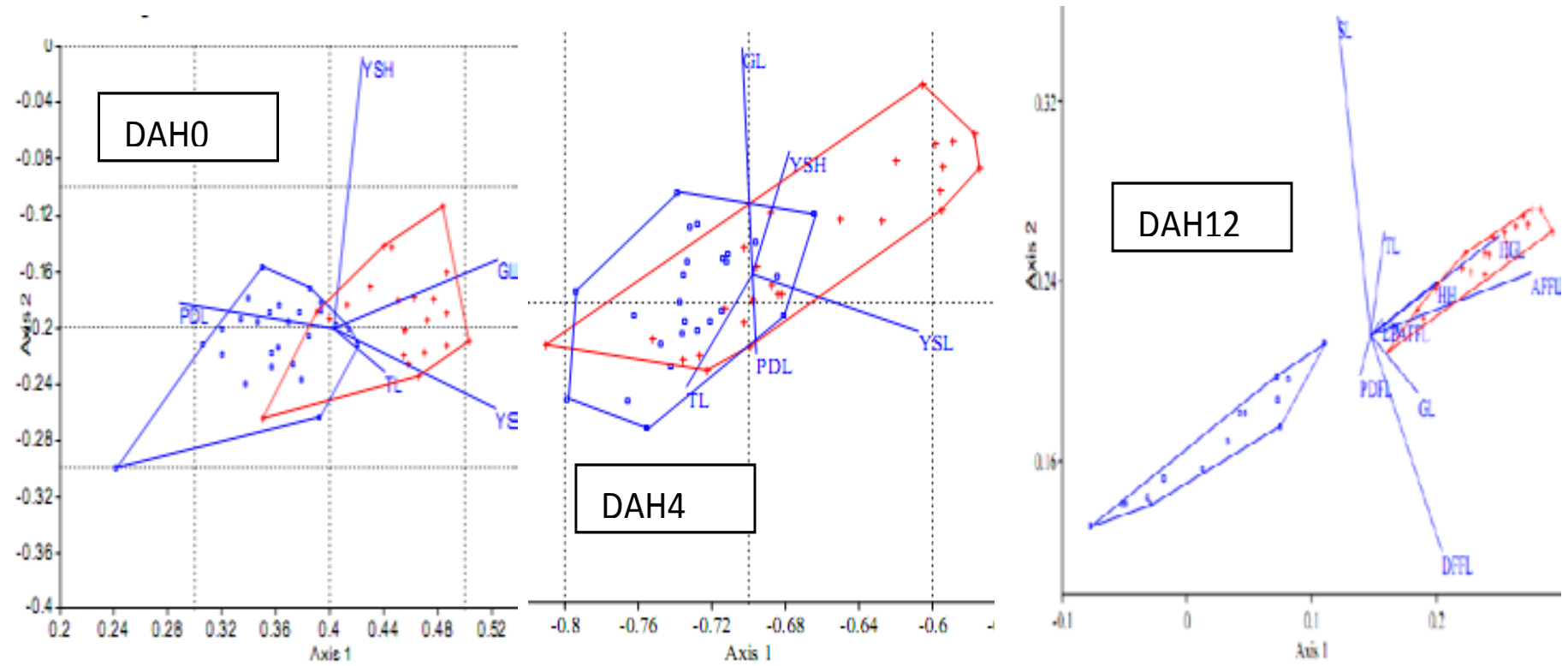

Figure 2. The CVA scatter plot for metric variables of the specimens $($ xenic $=$ red: + and axenic $=$ blue: $\square)$

\section{Analyses of shape}

While most of the variations in shape within groups of treatments at day zero post hatching are observed at head region by folding upward or downward (Figure $3 \mathrm{~A}$ and $\mathrm{B}$ ), in larvae of day four after hatching it involves variation in the height of the larvae fol- lowed by the bending at the head region (Figure $4 \mathrm{C}$ and D). In the age group of day 12 after hatching, PC1 reflects bending whereas PC2 larval height, with the bending being more prominent in axenic larvae (Figure $3 \mathrm{E}$ and $\mathrm{F}$ )

$$
\text { -2S.D. Mean +2S.D. }
$$

PC1
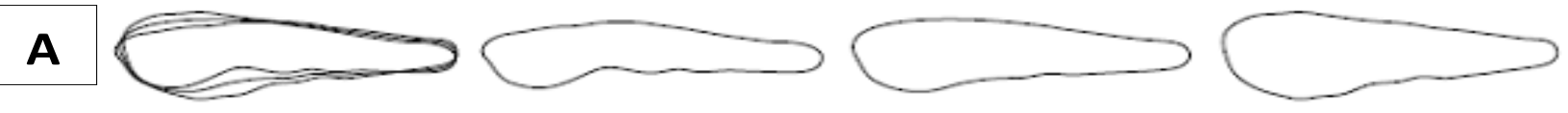

\section{B}
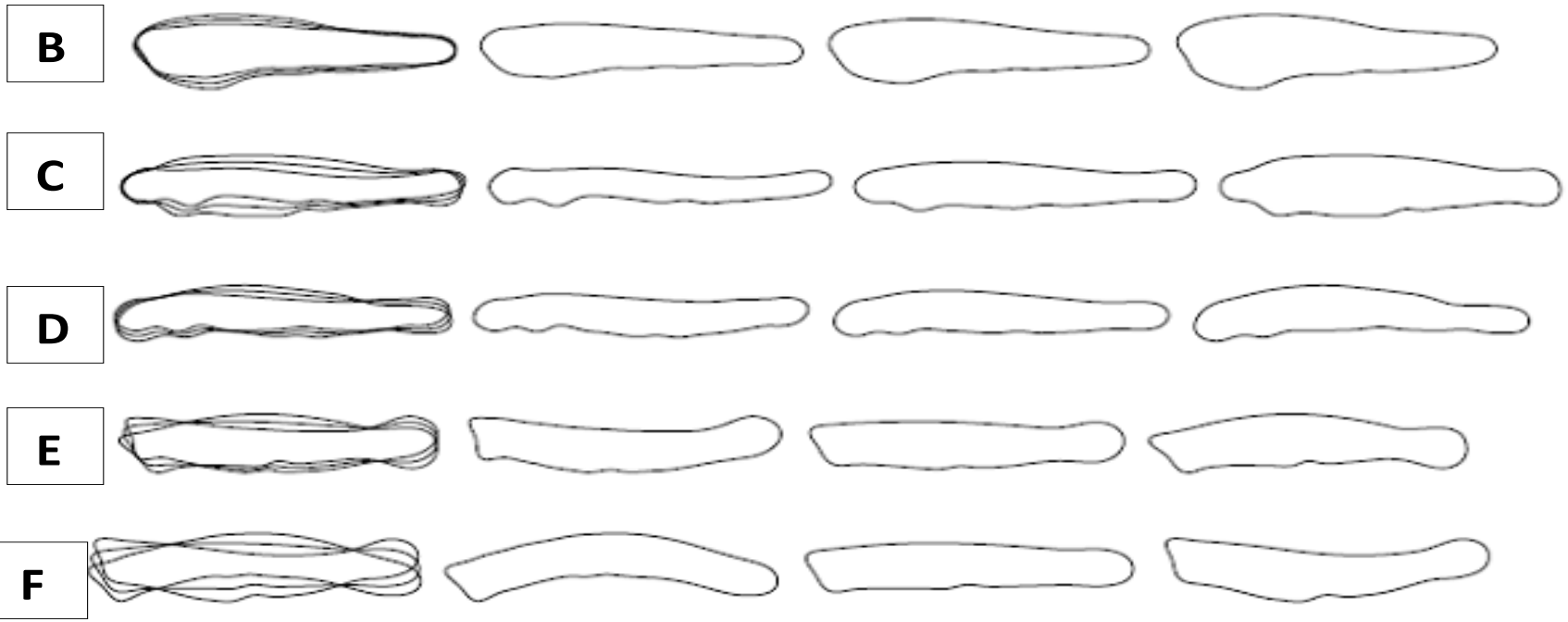

Figure 3. PC contours: $A=D A H 0-x e n i c, ~ B=D A H 0-a x e n i c, C=D A H 4-x e n i c, D=D A H 4-a x e n i c$, 


\section{DISCUSSION}

The average total length obtained in this study for xenic specimens were $3.67 \pm 0.14,4.39 \pm 0.34$ and $4.94 \pm 0.19 \mathrm{~mm}$ at day 0,4 and 12 after hatching, respectively (the axenic ones are significantly lower than xenic). These values are slightly smaller but comparable with previously reported total length. Saillant et al. (2001) reported the total length of 8-day-old larvae ranged between $4 \cdot 73$ and $5 \cdot 26$ $\mathrm{mm}$. Hatziathanasiou et al. (2002) reported a total length of $5.12 \pm 0.00 \mathrm{~mm}$ for a week old larvae after hatching; Anonymous (2004) about $4.5 \mathrm{~mm}$ at day 2 after hatching (this same author reported $4 \mathrm{~mm}$ total length for seabream at day 4 after hatching). The relatively smaller total length observed in this study may be due to low quality parents. Saillant et al. (2001) reported that egg size and total length were subjected to the influence of the female. These authors also noted that fertilization, survival during incubation and hatching were positively correlated and strongly influenced by the female parent and the interaction between both parents of the seabass.

In all the three age groups considered in this study, the gut length showed a strong positive correlation with respect to total length, except for the axenic larvae of day four after hatching. The growth pattern is nearly isometric. The difference in growth pattern of gut length of axenic larvae of DAH four may be attributed to the relative low resorption of yolk sac and concomitant low total length. The yolk sac size diminished successively as larvae gets older age. These indicate the decrease in yolk sac size was also evident with increasing age as there is no exogenous feeding and the larvae depend on yolk sac as source of food at early stage of growth. This is in line with the Maneewongsa and Tattanon (1982) hypothesis that the rate of resorption of the yolk is shown by decreasing diameter at older ages. According to Rønnestad (1998) the yolk is quickly resorbed during the embryonic and the early larval stages compared to later stages and
$95 \%$ is depleted by 100 hours post fertilization in the seabass. Thus, the yolk sac exhibited negatively allometric growth pattern.

\section{CONCLUSION}

Based on this study we conclude that the axenic rearing conditions render a better survival but a larger deformity (folding) incidence of larvae. The folding of larvae is prominent at older age of axenically treated ones as indicated by the PCA contours. The egg quality is most important for larval quality. In this study the low egg quality is evident from the observed low hatching rate and, later low survival of larvae and has resulted in higher bending of larvae. We suggest that the eggs are obtained from a single selected spawnners (not from wild as in this study) and same batch to compare the xenic and axenic conditions. Further investigations that consider more age groups from the same spawnners and the same batch is recommended to establish concrete knowledge about the effects of axenic rearing conditions on larval quality especially at later ages.

\section{ACKNOWLEDGMENTS}

I would like to gratefully acknowledge the invaluable cooperation of the staff of Evolutionary Morphology of Vertebrates, and Laboratory of Aquaculture and Artemia Reference Center, Ghent University. I am thankful to the Flemish Interuniversity Council (VLIR) for funding the study. 


\section{REFERENCES}

Dedi, J., Takeuchi, T., Seikai, T and Watanabe, T. (1995). Hypervitaminosis and safe levels of vitamin A for larval flounder (Paralichthys olivaceus) fed Artemia nauplii. Aquaculture 133: 135-146.

Divanach, P., Boglione, C., Menu, B., Koumoudouros, G., Kentouri, M and Cataudella S. (1996). Abnormalities in finfish mariculture: an overview of the problem, causes and solutions. In: Chantain, B., Saroglia, M.,Sweetman, J., Lavens, P. (Eds.). Seabass and seab-ream culture: Problem and prospects. International Workshop. Verona, Italy. October 16-18, 1996. European Aquaculture Society, Oostende, Belgium, 21pp.

FAO. (2007. Cultured Aquatic Species Information Program. http://www.fao.org/fi/website/FIRetrieveAction.do?dom $=$ culturespecies\&xml

Iwata, H. (2010). Elliptic Fourier version 1.3

Hatziathanasiou, A., Paspatis, M., Houbart, M., Kestemont, P., Stefanakis, S and Kentouri M. (20020. Survival, growth and feeding in early life stages of European sea bass (Dicentrarchus labrax) intensively cultured under different stocking densities. Aquaculture 205: 89-102.

Koumoundouros, G., Oran, G., Divanach, P., Stefanakis, S and Kentouri, M. (1997). The opercular complex deformity in intensive gilthead sea bream (Sparus aurata L.) larviculture: Moment of apparition and description. Aquaculture

\section{6:165-177}

Koumoundouros, G., Maingot, E., Divanach, P and Kentouri, M. (2002). Kyphosis in reared seabass (Dicentrarchus labrax L.): ontogeny and effects on mortality. Aquaculture 209: 49-58.

Nguyen, L.T.H and Janssen, C.R. (2002). Embryo-larval toxicity tests with the African catfish (Clarias gariepinus): Comparative sensitivity of endpoints. Archives of Environmental Contamination and Toxicology 42: 256-262.
Maneewongsa, S and Tattanon, T. (1982). Nature of eggs, larvae and juveniles of the seabass In: FAO Corporate Document Repository; Report of training course on seabass spawning and larval rearing songkhla, Thailand.

Polo, A., Yufera, M and Pascual, E., (1991). Effects of temperature on egg and larval development of Sparus aurata L. Aquaculture 92:367-375.

Rohlf, F.J. (2012). TPS file utility program version 1.60. http://life.bio.sunysb.edu/morph/.

Rønnestad, I., William, K., Amos, T., Mordechai, H and Hans J. F. (1998). Utilization of yolk fuels in developing eggs and larvae of European seabass (Dicentrarchus labrax). Aquaculture 162: 157-170.

Saillant, E., Chatain, B., Fostier, A., Przybyla, C and Fauvel, C. (2001). Parental influence on early development in the European seabass. Journal of Fish Biology 58: 1585-1600.

Sfakianakis, D.G., Koumoundouros, G., Divanach, P and Kentouri, M. (2004). Osteological development of the vertebral column and of the fins in Pagellus erythrinus (L. 1758): Temperature effect on the developmental plasticity and morpho-anatomical abnormalities. Aquaculture 232: 407-424.

Sfakianakis, D.G., Georgakopoulou, E., Papadakis, I.E., Divanach, P., Kentouri M and Koumoundouros, G. (2006). Environmental determinants of haemal lordosis in European seabass, Dicentrarchus labrax (Linnaeus, 1758). Aquaculture 254:54-64.

Verhaegen, Y., Dominique, A., Tania, De W., Philippe, D and and Patrick S. (2007). Deformities in larval gilthead seabream (Sparus aurata): A qualitative and quantitative analysis using geometric morphometrics. Aquaculture 268: 156-168. 